\title{
A CHARACTERIZATION OF WARPED PRODUCT PSEUDO-SLANT SUBMANIFOLDS IN NEARLY COSYMPLECTIC MANIFOLDS
}

\author{
REEM AL-GHEFARI and FALLEH R. AL-SOLAMY \\ Department of Mathematics \\ Faculty of Science \\ King Abdulaziz University \\ P. O. Box 80203 Jeddah 21589 \\ Saudi Arabia \\ e-mail: ralghefari@kau.edu.sa \\ falleh@hotmail.com
}

\begin{abstract}
Recently, the second author studied warped product pseudo-slant submanifolds of nearly cosymplectic manifolds and obtained the general inequality for the squared norm of the second fundamental form. In this paper, we obtain the necessary and sufficient conditions that a pseudo-slant submanifold to be a locally warped product manifold. We also provide a non-trivial example of warped product pseudo-slant submanifolds of almost contact metric manifolds.
\end{abstract}

\section{Introduction}

Almost contact manifolds with Killing structures tensors were defined in [3] as nearly cosymplectic manifolds. Later, these manifolds were studied by Blair and Showers from the topological point of view [5].

2010 Mathematics Subject Classification: 53C40, 53C42, 53C15.

Keywords and phrases: slant submanifold, pseudo-slant submanifold, nearly cosymplectic manifold, warped products.

Received May 10, 2017

(C) 2017 Scientific Advances Publishers 
An almost contact metric structure $(\varphi, \xi, \eta, g)$ satisfying $\left(\widetilde{\nabla}_{X} \varphi\right) X=0$ is called a nearly cosymplectic structure. If we consider $S^{5}$ as a totally geodesic hypersurface of a six dimensional sphere $S^{6}$, then it is known that $S^{5}$ has a non cosymplectic nearly cosymplectic structure. A normal nearly cosymplectic manifold is cosymplectic (see [4]).

On the other hand, pseudo-slant submanifolds of almost contact metric manifold were introduced by Carriazo [7] under the name antislant submanifolds. Later, the warped products of these submanifolds of Kaehler manifolds were studied by Sahin [11] under the name of warped product hemi-slant submanifolds. In this paper, we study pseudo-slant submanifolds and their warped products in a nearly cosymplectic manifold. We characterise that a pseudo-slant submanifold of a nearly cosymplectic manifold is locally a warped product submanifold under the obtained condition. Finally, we construct an example of warped product pseudo-slant submanifolds of an almost contact metric manifold.

\section{Preliminaries}

A $(2 n+1)$-dimensional $C^{\infty}$ manifold $\widetilde{M}$ is said to have an almost contact structure if there exist on $\widetilde{M}$ a tensor field $\varphi$ of type $(1,1)$, a vector field $\xi$ and a 1-form $\eta$ satisfying [5]

$$
\varphi^{2}=-I+\eta \otimes \xi, \quad \varphi \xi=0, \quad \eta \circ \varphi=0, \quad \eta(\xi)=1 .
$$

There always exists a Riemannian metric $g$ on an almost contact manifold $\widetilde{M}$ satisfying the following compatibility condition:

$$
\eta(X)=g(X, \xi), \quad g(\varphi X, \varphi Y)=g(X, Y)-\eta(X) \eta(Y),
$$

where $X$ and $Y$ are vector fields on $\widetilde{M}$ [5].

An almost contact structure $(\varphi, \xi, \eta)$ is said to be nearly cosymplectic if $\varphi$ is Killing, i.e., if

$$
\left(\widetilde{\nabla}_{X} \varphi\right) Y+\left(\widetilde{\nabla}_{Y} \varphi\right) X=0
$$


or any $X, Y$ tangent to $\widetilde{M}$, where $\widetilde{\nabla}$ denotes the Riemannian connection of the metric $g$. It is well known that an almost contact metric manifold is cosymplectic if and only if $\widetilde{\nabla} \varphi$ vanishes identically, i.e., $\left(\widetilde{\nabla}_{X} \varphi\right) Y=0$ and $\widetilde{\nabla}_{X} \xi=0$.

On a nearly cosymplectic manifold the structure vector field $\xi$ is Killing (see [9]), that is,

$$
g\left(\widetilde{\nabla}_{Y} \xi, Z\right)+g\left(\widetilde{\nabla}_{Z} \xi, Y\right)=0,
$$

for any $Y, Z$ tangent to $\widetilde{M}$.

Let $M$ be submanifold of an almost contact metric manifold $\widetilde{M}$ with induced metric $g$ and let $\nabla$ and $\nabla^{\perp}$ be the induced connections on the tangent bundle $T M$ and the normal bundle $T^{\perp} M$ of $M$, respectively. Denote by $\mathcal{F}(M)$ the algebra of smooth functions on $M$ and by $\Gamma(T M)$ the $\mathcal{F}(M)$-module of smooth sections of $T M$ over $M$. Then the Gauss and Weingarten formulas are given by
(a) $\widetilde{\nabla}_{X} Y=\nabla_{X} Y+h(X, Y)$;
(b) $\widetilde{\nabla}_{X} N=-A_{N} X+\nabla \frac{1}{X} N$,

for each $X, Y \in \Gamma(T M)$ and $N \in \Gamma\left(T^{\perp} M\right)$, where $h$ and $A_{N}$ are the second fundamental form and the shape operator (corresponding to the normal vector field $N$ ), respectively for the immersion of $M$ into $\widetilde{M}$. They are related as $g(h(X, Y), N)=g\left(A_{N} X, Y\right)$, where $g$ denotes the Riemannian metric on $\widetilde{M}$ as well as induced on $M$.

For any $X \in \Gamma(T M)$, we write

$$
\varphi X=T X+F X
$$

where $T X$ is the tangential component and $F X$ is the normal component of $\varphi X$. Similarly, if any $N \in \Gamma\left(T^{\perp} M\right)$, then $\varphi N$ decompose as

$$
\varphi N=B N+C N
$$


where $B N$ and $C N$ are the tangential and the normal components of $\varphi N$, respectively. A submanifold $M$ of an almost contact metric manifold $\widetilde{M}$ is said to be invariant if $F$ is identically zero, that is, $\varphi X \in \Gamma(T M)$ and antiinvariant if $T$ is identically zero, that is, $\varphi X \in \Gamma\left(T^{\perp} M\right)$, for any $X \in \Gamma(T M)$.

Now, if we denote by $\mathcal{P}_{X} Y$ and $Q_{X} Y$ the tangential and normal parts of $\left(\widetilde{\nabla}_{X} \varphi\right) Y$, then

$$
\left(\widetilde{\nabla}_{X} \varphi\right) Y=\mathcal{P}_{X} Y+Q_{X} Y
$$

for all $X, Y \in \Gamma(T M)$.

We refer to [13] for the properties of $\mathcal{P}$ and $Q$, which we enlist here for later use:

$$
\begin{aligned}
& \left(\mathrm{p}_{1}\right)\left(\text { i) } \quad \mathcal{P}_{X+Y} W=\mathcal{P}_{X} W+\mathcal{P}_{Y} W, \quad \text { (ii) } Q_{X+Y} W=Q_{X} W+Q_{Y} W\right. \\
& \left(\mathrm{p}_{2}\right)(\mathrm{i}) \mathcal{P}_{X}(Y+W)=\mathcal{P}_{X} Y+\mathcal{P}_{X} W, \quad \text { (ii) } Q_{X}(Y+W)=Q_{X} Y+Q_{X} W ; \\
& \left(\mathrm{p}_{3}\right)(\mathrm{i}) g\left(\mathcal{P}_{X} Y, W\right)=-g\left(Y, \mathcal{P}_{X} W\right), \text { (ii) } g\left(Q_{X} Y, N\right)=-g\left(Y, \mathcal{P}_{X} N\right) ; \\
& \left(\mathrm{p}_{4}\right) \mathcal{P}_{X} \varphi Y+Q_{X} \varphi Y=-\varphi\left(\mathcal{P}_{X} Y+Q_{X} Y\right),
\end{aligned}
$$

for all $X, Y, W \in \Gamma(T M)$ and $N \in \Gamma\left(T^{\perp} M\right)$.

Also, if $M$ is a submanifold of a nearly cosymplectic manifold $\widetilde{M}$, then from (2.3) and (2.8), we have

$$
\text { (a) } \mathcal{P}_{X} Y+\mathcal{P}_{Y} X=0 ; \text { (b) } Q_{X} Y+Q_{Y} X=0 \text {, }
$$

for any $X, Y \in \Gamma(T M)$.

As a generalisation of invariant and anti-invariant submanifolds Cabrerizo et al. [6] introduced the idea of slant submanifolds of almost contact metric manifolds in a similar way of Chen's characterization of slant submanifolds of almost Hermitian manifolds [8]. A submanifold $M$ 
of an almost contact metric manifold $\widetilde{M}$ is said to be a slant submanifold if for each non zero vector $X$ tangent to $M$ at $x$ the angle $\theta$ between $\varphi X$ and $T_{x} M$ is constant for all $X \in T_{x} M-\left\langle\xi_{x}\right\rangle$ and for any $x \in M$ [6]. Obviously if $\theta=0, M$ is invariant and if $\theta=\pi / 2, M$ is an antiinvariant submanifold. A slant submanifold is said to be proper slant if it is neither invariant nor anti-invariant submanifold. They proved the following characterization for a slant submanifold of an almost contact metric manifold.

Theorem 2.1 ([6]). Let $M$ be a submanifold of an almost contact metric manifold $\widetilde{M}$, such that $\xi$ is tangent to $M$. Then $M$ is slant if and only if there exists a constant $\lambda \in[0,1]$ such that

$$
T^{2}=\lambda(-I+\eta \otimes \xi)
$$

Furthermore, if $\theta$ is slant angle of $M$, then $\lambda=\cos ^{2} \theta$.

Following relations are straightforward consequences of the above theorem:

$$
\begin{aligned}
& g(T X, T Y)=\cos ^{2} \theta(g(X, Y)-\eta(Y) \eta(X)), \\
& g(F X, F Y)=\sin ^{2} \theta(g(X, Y)-\eta(Y) \eta(X)),
\end{aligned}
$$

for all $X, Y \in \Gamma(T M)$. We, also have the following chacterization for a slant submanifold $M$ of an almost contact metric manifold $\widetilde{M}$.

Theorem 2.2 ([15]). Let $M$ be a proper slant submanifold of an almost contact metric manifold $\widetilde{M}$, such that $\xi \in \Gamma(T M)$. Then

(a) $B F X=\sin ^{2} \theta(-X+\eta(X) \xi) ; \quad$ (b) $C F X=-F T X$, for any $X \in \Gamma(T M)$. 


\section{Pseudo-Slant Submanifolds and their Warped Products}

In [7], Carriazo introduced a new class of submanifolds known as pseudo-slant submanifolds of almost contact metric manifolds which are the generalizations of contact $\mathrm{CR}$ and slant submanifolds. He defined these submanifolds under the name anti-slant submanifolds as follows: A submanifold $M$ of an almost contact metric manifold $\widetilde{M}$ is said to be anti-slant (or pseudo-slant) if there exist two orthogonal distributions $D_{1}$ and $D_{2}$ satisfying:

(i) $T M=\mathcal{D}_{1} \oplus \mathcal{D}_{2} \oplus\langle\xi\rangle$;

(ii) $\mathcal{D}_{1}$ is an anti-invariant, i.e., $\varphi \mathcal{D}_{1} \subseteq T^{\perp} M$;

(iii) $\mathcal{D}_{2}$ is a slant distribution with slant angle $\theta \neq \frac{\pi}{2}$.

From the above definition it is clear that if $\theta=0$, then $M$ is a contact CR-submanifold and if we consider the dimensions of $\mathcal{D}_{1}$ and $\mathcal{D}_{2}$ by $q$ and $p$, respectively. Then, if $q=0$ (respectively, $p=0$ ), then $M$ is proper slant (respectively, anti-invariant). Also, if $q=0$ and $\theta=0$, then $M$ is an invariant submanifold of $\widetilde{M}$.

If $\nu$ is an invariant normal subbundle of the normal bundle $T^{\perp} M$ of a pseudo-slant submanifold $M$, then we have

$$
T^{\perp} M=\varphi \mathcal{D}_{1} \oplus F \mathcal{D}_{2} \oplus \nu .
$$

Now, we have the following results for later use on a pseudo-slant submanifold $M$ of a nearly cosymplectic manifold $\widetilde{M}$.

Theorem 3.1. Let $M$ be a pseudo-slant submanifold of a nearly cosymplectic manifold $\widetilde{M}$, then

$$
\begin{aligned}
g\left(\nabla_{X} Y, Z\right)= & \sec ^{2} \theta g\left(A_{\varphi Z} T Y-A_{F T Y} Z, X\right)+\tan ^{2} \theta \eta(Y) g\left(\nabla_{Z} \xi, X\right) \\
& -\sec ^{2} \theta g\left(Q_{X} Y, \varphi Z\right)-\sec ^{2} \theta g\left(Q_{X} Z, F Y\right)
\end{aligned}
$$

for any $X, Y \in \Gamma\left(\mathcal{D}_{2} \oplus\langle\xi\rangle\right)$ and any $Z \in \Gamma\left(\mathcal{D}_{1}\right)$. 
Proof. For any $X, Y \in \Gamma\left(\mathcal{D}_{2} \oplus\langle\xi\rangle\right)$ and $Z \in \Gamma\left(\mathcal{D}_{1}\right)$, we have

$$
\begin{aligned}
g\left(\widetilde{\nabla}_{X} Y, Z\right) & =g\left(\varphi \widetilde{\nabla}_{X} Y, \varphi Z\right) \\
& =g\left(\widetilde{\nabla}_{X} \varphi Y, \varphi Z\right)-g\left(\left(\widetilde{\nabla}_{X} \varphi\right) Y, \varphi Z\right) .
\end{aligned}
$$

Using (2.6) and (2.8), we get

$$
g\left(\nabla_{X} Y, Z\right)=g\left(\widetilde{\nabla}_{X} T Y, \varphi Z\right)+g\left(\widetilde{\nabla}_{X} F Y, \varphi Z\right)-g\left(Q_{X} Y, F Z\right) .
$$

Then from (2.2) and (2.5) (a), we obtain

$$
g\left(\nabla_{X} Y, Z\right)=g(h(X, T Y) \varphi Z)-g\left(\varphi \widetilde{\nabla}_{X} F Y, Z\right)-g\left(Q_{X} Y, F Z\right) .
$$

Using the covariant differentiation property of $\varphi$, we derive

$$
\begin{aligned}
g\left(\nabla_{X} Y, Z\right)= & g(h(X, T Y) \varphi Z)+g\left(\left(\widetilde{\nabla}_{X} \varphi\right) F Y, Z\right)-g\left(\widetilde{\nabla}_{X} \varphi F Y, Z\right) \\
& -g\left(Q_{X} Y, F Z\right) \\
= & g\left(A_{\varphi Z} T Y, X\right)+g\left(\mathcal{P}_{X} F Y, Z\right)-g\left(\widetilde{\nabla}_{X} B F Y, Z\right) \\
& -g\left(\tilde{\nabla}_{X} C F Y, Z\right)-g\left(Q_{X} Y, F Z\right) .
\end{aligned}
$$

From the property $\left(\mathrm{p}_{3}\right)$ (ii) and the Theorem 2.2 , we obtain

$$
\begin{aligned}
g\left(\nabla_{X} Y, Z\right)= & g\left(A_{\varphi Z} T Y, X\right)-g\left(Q_{X} Z, F Y\right)+\sin ^{2} \theta g\left(\widetilde{\nabla}_{X} Y, Z\right) \\
& -\eta(Y) \sin ^{2} \theta g\left(\widetilde{\nabla}_{X} \xi, Z\right)+g\left(\widetilde{\nabla}_{X} F T Y, Z\right)-g\left(Q_{X} Y, F Z\right) .
\end{aligned}
$$

Then by (2.4) and (2.5), we arrive at

$$
\begin{aligned}
\cos ^{2} \theta g\left(\nabla_{X} Y, Z\right)= & g\left(A_{\varphi Z} T Y, X\right)-g\left(A_{F T Y} X, Z\right)+\eta(Y) \sin ^{2} \theta g\left(\tilde{\nabla}_{Z} \xi, X\right) \\
& -g\left(Q_{X} Z, F Y\right)-g\left(Q_{X} Y, F Z\right) .
\end{aligned}
$$

Thus, the proof follows from the last relation by using the symmetry of the shape operator $A$.

We have the following corollary as a consequence of the above theorem. 
Corollary 3.1. Let $M$ be a pseudo-slant submanifold of a nearly cosymplectic manifold $\widetilde{M}$, then the slant distribution $\mathcal{D}_{2} \oplus\langle\xi\rangle$ defines a totally geodesic foliation on $M$ if and only if

$$
g\left(A_{F T Y} Z-A_{\varphi Z} T Y, X\right)=\sin ^{2} \theta \eta(Y) g\left(\nabla_{Z} \xi, X\right) \text { and } Q_{X} U \in \nu,
$$

for any $X, Y \in \Gamma\left(\mathcal{D}_{2} \oplus\langle\xi\rangle\right)$, any $Z \in \Gamma\left(\mathcal{D}_{1}\right)$ and any $U \in \Gamma(T M)$.

Theorem 3.2. Let $M$ be a pseudo-slant submanifold of a nearly cosymplectic manifold $\widetilde{M}$, then

$$
\begin{aligned}
\cos ^{2} \theta g([Z, W], X)=g\left(A_{\varphi Z} W-A_{\varphi W} Z, T X\right) & -2 \cos ^{2} \theta \eta(X) g\left(\nabla_{Z} \xi, W\right) \\
& -2 g\left(\mathcal{P}_{Z} W, T X\right)
\end{aligned}
$$

for any $Z, W \in \Gamma\left(\mathcal{D}_{1}\right)$ and any $X \in \Gamma\left(\mathcal{D}_{2} \oplus\langle\xi\rangle\right)$.

Proof. For any $Z, W \in \Gamma\left(\mathcal{D}_{1}\right)$ and $X \in \Gamma\left(\mathcal{D}_{2} \oplus\langle\xi\rangle\right)$, we have

$$
\begin{aligned}
g([Z, W], X)= & g\left(\widetilde{\nabla}_{Z} W, X\right)-g\left(\widetilde{\nabla}_{W} Z, X\right) \\
= & g\left(\varphi \widetilde{\nabla}_{Z} W, \varphi X\right)-g\left(\varphi \widetilde{\nabla}_{W} Z, \varphi X\right)+\eta(X) g\left(\widetilde{\nabla}_{Z} W, \xi\right) \\
& -\eta(X) g\left(\widetilde{\nabla}_{W} Z, \xi\right) .
\end{aligned}
$$

Then by the covariant derivative property of $\varphi$, we get

$$
\begin{aligned}
g([Z, W], X)= & g\left(\widetilde{\nabla}_{Z} \varphi W, \varphi X\right)-g\left(\left(\widetilde{\nabla}_{Z} \varphi\right) W, \varphi X\right)-g\left(\widetilde{\nabla}_{W} \varphi Z, \varphi X\right) \\
& +g\left(\left(\widetilde{\nabla}_{W} \varphi\right) Z, \varphi X\right)-\eta(X) g\left(\widetilde{\nabla}_{Z} \xi, W\right)+\eta(X) g\left(\widetilde{\nabla}_{W} \xi, Z\right) .
\end{aligned}
$$

By the orthogonality of vector fields and using (2.4), (2.6), and (2.8), we obtain

$$
\begin{aligned}
g([Z, W], X)= & -g\left(\varphi W, \tilde{\nabla}_{Z} \varphi X\right)-g\left(\mathcal{P}_{Z} W, T X\right)-g\left(Q_{Z} W, F X\right) \\
& -g\left(\varphi Z, \widetilde{\nabla}_{W} \varphi X\right)+g\left(\mathcal{P}_{W} Z, T X\right)+g\left(Q_{W} Z, F X\right) \\
& -2 \eta(X) g\left(\widetilde{\nabla}_{Z} \xi, W\right) .
\end{aligned}
$$

Again using (2.6), (2.9), (2.2), (2.5) and the covariant derivative of $\varphi$, we derive 


$$
\begin{aligned}
g([Z, W], X)= & -g(\varphi W, h(Z, T X))+g\left(\widetilde{\nabla}_{Z} \varphi F X, W\right)-g\left(\left(\widetilde{\nabla}_{Z} \varphi\right) F X, W\right) \\
& -2 g\left(\mathcal{P}_{Z} W, T X\right)-2 g\left(Q_{Z} W, F X\right)+g(\varphi Z, h(W, T X)) \\
& -g\left(\widetilde{\nabla}_{W} \varphi F X, Z\right)+g\left(\left(\widetilde{\nabla}_{W} \varphi\right) F X, Z\right)-2 \eta(X) g\left(\widetilde{\nabla}_{Z} \xi, W\right) . \\
= & g\left(A_{\varphi Z} W-A_{\varphi W} Z, T X\right)+g\left(\widetilde{\nabla}_{Z} B F X, W\right)+g\left(\widetilde{\nabla}_{Z} C F X, W\right) \\
& -g\left(\mathcal{P}_{Z} F X, W\right)-2 g\left(\mathcal{P}_{Z} W, T X\right)-2 g\left(Q_{Z} W, F X\right) \\
& -g\left(\widetilde{\nabla}_{W} B F X, Z\right)-g\left(\widetilde{\nabla}_{W} C F X, Z\right)+g\left(\mathcal{P}_{W} F X, Z\right) \\
& -2 \eta(X) g\left(\widetilde{\nabla}_{Z} \xi, W\right) .
\end{aligned}
$$

From the property $\left(\mathrm{p}_{3}\right)$ (ii) and the Theorem 2.2, we arrive at

$$
\begin{aligned}
g([Z, W], X)= & g\left(A_{\varphi Z} W-A_{\varphi W} Z, T X\right)-\sin ^{2} \theta g\left(\widetilde{\nabla}_{Z} X, W\right)+g\left(Q_{Z} W, F X\right) \\
& +\sin ^{2} \theta \eta(X) g\left(\widetilde{\nabla}_{Z} \xi, W\right)-g\left(\widetilde{\nabla}_{Z} F T X, W\right)-g\left(Q_{W} Z, F X\right) \\
& -2 g\left(\mathcal{P}_{Z} W, T X\right)-2 g\left(Q_{Z} W, F X\right)+\sin ^{2} \theta g\left(\widetilde{\nabla}_{W} X, Z\right) \\
& -\sin ^{2} \theta \eta(X) g\left(\widetilde{\nabla}_{W} \xi, Z\right)+g\left(\widetilde{\nabla}_{W} F T X, Z\right)-2 \eta(X) g\left(\widetilde{\nabla}_{Z} \xi, W\right) .
\end{aligned}
$$

Using the orthogonality of distributions and (2.4), (2.5), we obtain

$$
\begin{aligned}
g([Z, W], X)= & g\left(A_{\varphi Z} W-A_{\varphi W} Z, T X\right)+\sin ^{2} \theta g\left(\widetilde{\nabla}_{Z} W, X\right) \\
& +2 \sin ^{2} \theta \eta(X) g\left(\widetilde{\nabla}_{Z} \xi, W\right)+g\left(A_{F T X} Z, W\right)-2 g\left(\mathcal{P}_{Z} W, T X\right) \\
& -\sin ^{2} \theta g\left(\widetilde{\nabla}_{W} Z, X\right)-g\left(A_{F T X} W, Z\right)-2 \eta(X) g\left(\widetilde{\nabla}_{Z} \xi, W\right) .
\end{aligned}
$$

By the symmetry of the shape operator $A$, we derive

$$
\begin{aligned}
\cos ^{2} \theta g([Z, W], X)= & g\left(A_{\varphi Z} W-A_{\varphi W} Z, T X\right)-2 \cos ^{2} \theta \eta(X) g\left(\nabla_{Z} \xi, W\right) \\
& -2 g\left(\mathcal{P}_{Z} W, T X\right)
\end{aligned}
$$

which is (3.2), hence the proof is complete. 
In [1], Al-Solamy obtained the following results which are useful to prove the characterization of warped products. He also derived an inequality for the second fundamental form of the warped product pseudo-slant submanifold of the type $M=M_{\theta} \times_{f} M_{\perp}$ of a nearly cosymplectic manifold $\widetilde{M}$ such that the structure vector field $\xi$ tangent to $M_{\theta}$.

Lemma 3.1 ([1]). Let $M=M_{\theta} \times_{f} M_{\perp}$ be a warped product pseudoslant submanifold of a nearly cosymplectic manifold $\widetilde{M}$, then

(i) $\xi \ln f=0$;

(ii) $2 g(h(X, Y), \varphi Z)=g(h(X, Z), F Y)+g(h(Y, Z), F X)$, for any $X, Y \in \Gamma\left(T M_{\theta}\right)$ and $Z \in \Gamma\left(T M_{\perp}\right)$.

Lemma 3.2 ([1]). Let $M=M_{\theta} \times_{f} M_{\perp}$ be a warped product pseudoslant submanifold of a nearly cosymplectic manifold $\widetilde{M}$, where $M_{\perp}$ and $M_{\theta}$ are anti-invariant and proper slant submanifolds of $\widetilde{M}$, respectively. Then,

$$
\begin{aligned}
2 g(h(Z, W), F T X)= & g(h(T X, Z), \varphi W)+g(h(T X, W), \varphi Z) \\
& -2 \cos ^{2} \theta(X \ln f) g(Z, W),
\end{aligned}
$$

for any $X \in \Gamma\left(T M_{\theta}\right)$ and $Z, W \in \Gamma\left(T M_{\perp}\right)$.

Now, we are able to prove the main result of this section.

Theorem 3.3. Let $M$ be a pseudo-slant submanifold of a nearly cosymplectic manifold $\widetilde{M}$ such that the normal component of $\left(\widetilde{\nabla}_{U} \varphi\right) V$ lies in the invariant normal subbundle of $M$, i.e., $Q_{U} V \in \Gamma(\nu)$, for any $U, V \in \Gamma(T M)$ and the tangential component of $\left(\widetilde{\nabla}_{Z} \varphi\right) W$ lies in the anti- 
invariant distribution, i.e., $\mathcal{P}_{Z} W \in \Gamma\left(\mathcal{D}_{1}\right)$, for any $Z, W \in \Gamma\left(\mathcal{D}_{1}\right)$. Then $M$ is locally a mixed geodesic warped product submanifold if and only if

$$
A_{\varphi Z} X=0, \quad A_{F T X} Z=-\cos ^{2} \theta(X \mu) Z, \quad \text { and } \quad g\left(\nabla_{Z} \xi, U\right)=0 \text {, }
$$

for any $X \in \Gamma\left(\mathcal{D}_{2} \oplus\langle\xi\rangle\right), Z \in \Gamma\left(\mathcal{D}_{1}\right)$ and $U \in \Gamma(T M)$, where $\mu$ is a smooth function on $M$ such that $W(\mu)=0$, for any $W \in \Gamma\left(\mathcal{D}_{1}\right)$.

Proof. If $M$ is a mixed geodesic warped product pseudo-slant submanifold of the form $M=M_{\theta} \times_{f} M_{\perp}$ of a nearly cosymplectic manifold $\widetilde{M}$, then first two parts of (3.3) directly follow from Lemma 3.1 (ii) and Lemma 3.2. Also, $g\left(\nabla_{Z} \xi, U\right)=\xi \ln f g(Z, U)=0$ (using Lemma 3.1 (i)), for any $Z \in \Gamma\left(T M_{\perp}\right)$ and any $U \in \Gamma(T M)$.

Conversely, if $M$ is a pseudo-slant submanifold with the given conditions such that (3.3) holds, then from (3.1), we have

$$
\begin{aligned}
\cos ^{2} \theta g\left(\nabla_{X} Y, Z\right)= & g\left(A_{\varphi Z} X, T Y\right)-g\left(A_{F T Y} Z, X\right)+\sin ^{2} \theta \eta(Y) g\left(\nabla_{Z} \xi, X\right) \\
& -g\left(Q_{X} Y, \varphi Z\right)-g\left(Q_{X} Z, F Y\right)
\end{aligned}
$$

Then from (3.3) and by the given fact that $Q_{U} V \in \Gamma(\nu)$, for any $U, V \in \Gamma(T M)$, we get $\cos ^{2} \theta g\left(\nabla_{X} Y, Z\right)=0$, since $M$ is proper pseudoslant then $\theta \neq \pi / 2$ thus the leaves of $\mathcal{D}_{2} \oplus\langle\xi\rangle$ are totally geodesic in $M$. On the other hand, from (3.2), we have

$$
\begin{aligned}
\cos ^{2} \theta g([Z, W], X)= & g\left(A_{\varphi Z} T X, W\right)-g\left(A_{\varphi W} T X, Z\right) \\
& -2 \cos ^{2} \theta \eta(X) g\left(\nabla_{Z} \xi, W\right)-2 g\left(\mathcal{P}_{Z} W, T X\right),
\end{aligned}
$$

for any $Z, W \in \Gamma\left(\mathcal{D}_{1}\right)$ and any $X \in \Gamma\left(\mathcal{D}_{2} \oplus\langle\xi\rangle\right)$. Using (3.3) and the fact that $Q_{U} V \in \Gamma(\nu)$, for any $U, V \in \Gamma(T M)$ and $\mathcal{P}_{Z} W \in \Gamma\left(\mathcal{D}_{1}\right)$, for any $Z, W \in \Gamma\left(\mathcal{D}_{1}\right)$, we get $\cos ^{2} \theta g([Z, W], X)=0$. Since $M$ is proper slant then $\cos \theta \neq 0$ thus we conclude that $\mathcal{D}_{1}$ is integrable. If we consider a 
leaf $M_{\perp}$ of $\mathcal{D}_{1}$ in $M$ with second fundamental form $\hat{h}$ of $M_{\perp}$ in $M$, then for any $Z, W \in \Gamma\left(\mathcal{D}_{1}\right)$ and $X \in \Gamma\left(\mathcal{D}_{2} \oplus\langle\xi\rangle\right)$, we have

$$
g(\hat{h}(Z, W), X)=g\left(\nabla_{Z} W, X\right)=g\left(\widetilde{\nabla}_{Z} W, X\right) .
$$

From (2.2), we obtain

$$
\begin{aligned}
g(\hat{h}(Z, W), X) & =g\left(\varphi \widetilde{\nabla}_{Z} W, \varphi X\right)+\eta(X) g\left(\widetilde{\nabla}_{Z} W, \xi\right) \\
& =g\left(\widetilde{\nabla}_{Z} \varphi W, \varphi X\right)-g\left(\left(\widetilde{\nabla}_{Z} \varphi\right) W, \varphi X\right)-\eta(X) g\left(\nabla_{Z} \xi, W\right) .
\end{aligned}
$$

Again using (2.2), (2.6), (2.8) and the last term of (3.3), we arrive at

$$
\begin{aligned}
g(\hat{h}(Z, W), X)= & -g\left(\varphi W, \tilde{\nabla}_{Z} T X\right)-g\left(\varphi W, \tilde{\nabla}_{Z} F X\right) \\
& -g\left(\mathcal{P}_{Z} W, T X\right)-g\left(Q_{Z} W, F X\right) .
\end{aligned}
$$

Then by the hypothesis of the theorem, the last two terms of the right hand side are identically zero. Thus, on using (2.2), (2.5) and the covariant derivative property of $\varphi$, we derive

$$
\begin{aligned}
g(\hat{h}(Z, W), X)= & -g(\varphi W, h(Z, T X))+g\left(\widetilde{\nabla}_{Z} \varphi F X, W\right)-g\left(\left(\widetilde{\nabla}_{Z} \varphi\right) F X, W\right) \\
= & -g\left(A_{\varphi W} T X, Z\right)+g\left(\widetilde{\nabla}_{Z} B F X, W\right)+g\left(\widetilde{\nabla}_{Z} C F X, W\right) \\
& -g\left(\mathcal{P}_{Z} F X, W\right) .
\end{aligned}
$$

Hence, the first term of the right and side is identically zero by using the first part of (3.3), then from the property $\left(\mathrm{p}_{3}\right)$ (ii) and Theorem 2.2 , we obtain

$$
\begin{aligned}
g(\hat{h}(Z, W), X)= & -\sin ^{2} \theta g\left(\tilde{\nabla}_{Z} X, W\right)+\sin ^{2} \theta \eta(X) g\left(\tilde{\nabla}_{Z} \xi, W\right) \\
& -g\left(\tilde{\nabla}_{Z} F T X, W\right)+g\left(Q_{Z} W, F X\right) .
\end{aligned}
$$

By the hypothesis of the theorem, the second and the last terms in the right hand side vanish identically. Then, we get

$$
g(\hat{h}(Z, W), X)=\sin ^{2} \theta g\left(\tilde{\nabla}_{Z} W, X\right)+g\left(A_{F T X} Z, W\right) .
$$


Then, from the second part of (3.3), we derive

$$
\cos ^{2} \theta g(\hat{h}(Z, W), X)=-\cos ^{2} \theta(X \mu) g(Z, W),
$$

or

$$
\hat{h}(Z, W)=-\vec{\nabla} \mu g(Z, W),
$$

which shows that $M_{\perp}$ is a totally umbilical submanifold of $M$ with mean curvature $\hat{H}=-\vec{\nabla} \mu$, where $\vec{\nabla} \mu$ is the gradient of the function $\mu$. Furthermore, $W(\mu)=0$, for any $W \in \Gamma\left(\mathcal{D}_{1}\right)$ implies that $\hat{H}$ is parallel mean curvature vector. Then by a result of Hiepko [10], we conclude that $M$ is locally a warped product manifold, which completes the proof.

Example 3.1. Consider a submanifold of $\mathbb{R}^{9}$ such that the Cartesian coordinates of $\mathbb{R}^{9}$ are $\left(x_{1}, x_{2}, x_{3}, x_{4}, y_{1}, y_{2}, y_{3}, y_{4}, t\right)$ and the almost contact structure is given by

$$
\varphi\left(\frac{\partial}{\partial x_{i}}\right)=-\frac{\partial}{\partial y_{i}}, \quad \varphi\left(\frac{\partial}{\partial y_{j}}\right)=\frac{\partial}{\partial x_{j}}, \quad \varphi\left(\frac{\partial}{\partial t}\right)=0, \quad 1 \leq i, j \leq 4 .
$$

If $X=\lambda_{i} \frac{\partial}{\partial x_{i}}+\mu j \frac{\partial}{\partial y_{j}}+\nu \frac{\partial}{\partial t}$ is a vector field of $\mathbb{R}^{9}$, then $\varphi X=-\lambda_{i} \frac{\partial}{\partial y_{i}}$ $+\mu_{j} \frac{\partial}{\partial x_{j}}$ and $\varphi^{2}(X)=-\lambda_{i} \frac{\partial}{\partial x_{i}}-\mu_{j} \frac{\partial}{\partial y_{j}}=-X+\nu \frac{\partial}{\partial t}$. Also, we can see that $g(X, X)=\lambda_{i}^{2}+\mu_{j}^{2}+\nu^{2}$ and $g(\varphi X, \varphi X)=\lambda_{i}^{2}+\mu_{j}^{2}$, where $g$ is the Euclidean metric tensor of $\mathbb{R}^{9}$. Then, we have $g(\varphi X, \varphi X)=g(X, X)-\eta(X) \xi$, where $\xi=\frac{\partial}{\partial t}$ and hence $(\varphi, \xi, \eta, g)$ is an almost contact structure on $\mathbb{R}^{9}$. Now consider a submanifold $M$ of $\mathbb{R}^{9}$ defined by the immersion

$$
\chi(u, v, w, s, t)=\left(-u \cos \theta, u \sin \theta, e^{t} w, 0, v \cos \theta, v \sin \theta, 0, e^{t} s, t\right),
$$

for any $\theta \neq 0, \frac{\pi}{4}$. Then, the tangent space $T M$ of $M$ is spanned by the following vectors: 


$$
\begin{gathered}
Z_{1}=-\cos \theta \frac{\partial}{\partial x_{1}}+\sin \theta \frac{\partial}{\partial x_{2}}, \quad Z_{2}=\cos \theta \frac{\partial}{\partial y_{1}}+\sin \theta \frac{\partial}{\partial y_{2}}, \\
Z_{3}=e^{t} \frac{\partial}{\partial x_{3}}, \quad Z_{4}=e^{t} \frac{\partial}{\partial y_{4}}, \quad Z_{5}=\frac{\partial}{\partial t} .
\end{gathered}
$$

Thus, we have

$$
\begin{gathered}
\varphi Z_{1}=\cos \theta \frac{\partial}{\partial y_{1}}-\sin \theta \frac{\partial}{\partial y_{2}}, \quad \varphi Z_{2}=\cos \theta \frac{\partial}{\partial x_{1}}+\sin \theta \frac{\partial}{\partial x_{2}}, \\
\varphi Z_{3}=-e^{t} \frac{\partial}{\partial y_{3}}, \quad \varphi Z_{4}=e^{t} \frac{\partial}{\partial x_{4}}, \quad \varphi Z_{5}=0 .
\end{gathered}
$$

Clearly, the vector fields $\varphi Z_{3}$ and $\varphi Z_{4}$ are orthogonal to $T M$. Then the anti-invariant and proper slant distributions of $M$, respectively, are $\mathcal{D}_{1}=\operatorname{Span}\left\{Z_{3}, Z_{4}\right\}$ and $\mathcal{D}_{2}=\operatorname{Span}\left\{Z_{1}, Z_{2}\right\}$ with slant angle $\theta_{1}=\cos ^{-1}$ $\left(\frac{g\left(Z_{1}, \varphi Z_{2}\right)}{\left\|Z_{1}\right\|\left\|\varphi Z_{2}\right\|}\right)=\cos ^{-1}\left(\frac{g\left(Z_{2}, \varphi Z_{1}\right)}{\left\|Z_{2}\right\|\left\|\varphi Z_{1}\right\|}\right)=2 \theta$ such that $\xi=Z_{5}$ is tangent to $M$.

Hence, $M$ is a proper pseudo-slant submanifold of $\mathbb{R}^{9}$. Furthermore, it is easy to se that $\mathcal{D}_{1}$ and $\mathcal{D}_{2}$ are integrable. We denote the integral manifolds of $\mathcal{D}_{1}$ and $\mathcal{D}_{2}$ by $M_{\perp}$ and $M_{\theta}$, respectively. Then the metric tensor $g$ of $M$ is

$$
g=d u^{2}+d v^{2}+d t^{2}+e^{2 t}\left(d w^{2}+d s^{2}\right)=g_{1}+e^{2 t} g_{2}
$$

Thus $M$ is a warped product pseudo-slant submanifold of $\mathbb{R}^{9}$ with warping function $f=e^{t}$ such that $Z_{5}=\xi$ is tangent to $M_{\theta}$.

\section{References}

[1] F. R. Al-Solamy, An inequality for warped product pseudo-slant submanifolds of nearly cosymplectic manifolds, J. Inequal. Appl. 306 (2015), 1-9.

[2] R. L. Bishop and B. O’Neill, Manifolds of negative curvature, Trans. Amer. Math. Soc. 145 (1969), 1-49.

[3] D. E. Blair, Almost contact manifolds with Killing structure tensors I, Pac. J. Math. 39 (1971), 285-292. 
[4] D. E. Blair and K. Yano, Affine almost contact manifolds and $f$-manifolds with affine killing structure tensors, Kodai Math. Sem. Rep. 23 (1971), 473-479.

[5] D. E. Blair and D. K. Showers, Almost contact manifolds with Killing structures tensors II, J. Differ. Geom. 9 (1974), 577-582.

[6] J. L. Cabrerizo, A. Carriazo, L. M. Fernandez and M. Fernandez, Slant submanifolds in Sasakian manifolds, Glasgow Math. J. 42 (2000), 125-138.

[7] A. Carriazo, New Developments in Slant Submanifolds Theory, Narosa Publishing House, New Delhi, India, 2002.

[8] B. -Y. Chen, Slant immersions, Bull. Austral. Math. Soc. 41 (1990), 135-147.

[9] H. Endo, On the curvature tensor of nearly cosymplectic manifolds of constant $\varphi$-sectional curvature, Ann. St. Univ. Iasi. Tom. LI $f_{2}$ (2005), 439-554.

[10] S. Hiepko, Eine inner kennzeichungder verzerrten produkte, Math. Ann. 241 (1979), 209-215.

[11] B. Sahin, Warped product submanifolds of Kaehler manifolds with a slant factor, Ann. Pol. Math. 95 (2009), 207-226.

[12] S. Uddin, S. H. Kon, M. A. Khan and Khushwant Singh, Warped product semiinvariant submanifolds of nearly cosymplectic manifolds, Math. Probl. Eng. 2011 (2011).

[13] S. Uddin, A. Mustafa, B. R. Wong and C. Ozel, A geometric inequality for warped product semi-slant submanifolds of nearly cosymplectic manifolds, Rev. Dela Union Math. Argentina 55 (2014), 55-69.

[14] S. Uddin and F. R. Al-Solamy, Warped product pseudo-slant submanifolds of cosymplectic manifolds, An. Ştiinț. Univ. Al. I. Cuza Iaşi Mat. (N. S.) Tome LXIII $f_{2}$ 3 (2016), 901-913.

[15] S. Uddin and F. R. Al-Solamy, Warped product pseudo-slant immersions in Sasakian manifolds, Publ. Math. Debrecen 91(2) (2017), 1-14. 